\title{
Malignant lymphoma presenting with a high eosinophilia, eosinophilic pleurisy, and pericarditis
}

\author{
A. H. HENDERSON AND G. MEJ I A ${ }^{1}$ \\ From the Royal Devon and Exeter Hospital, Exeter
}

\begin{abstract}
Two cases of malignant lymphoma with an unusually high eosinophilia are reported. The first patient presented with eosinophilic pleural effusion, developed eosinophilic pericarditis and tamponade, and was found at thoracotomy to have lymphosarcoma. The occurrence in malignant lymphoma of these uncommon features is briefly reviewed. The development of a pleurobronchial fistula is also described as an alarming complication of pleural paracentesis.
\end{abstract}

A high eosinophilia, eosinophilic pleurisy, and eosinophilic pericarditis are all uncommon. Malignant lymphoma is not readily considered among their differential diagnosis. One patient with lymphosarcoma who presented with these three features and one patient with Hodgkin's disease and a high eosinophilia are reported.

\section{CASE REPORTS}

CASE 1 A previously fit 19-year-old ship's cook, who had not visited the tropics, was dyspnoeic for two weeks before admission on 7 November 1966 to the Royal South Hants Hospital. He was transferred on 25 November to the Royal Devon and Exeter Hospital, and was found to have a large right pleural effusion, but no other abnormal signs and no pyrexia.

The haemoglobin was $14.5 \mathrm{~g} . / 100 \mathrm{ml}$., white blood cell count 18,300 , with 8,200 eosinophils/cu. mm., and 160,000 platelets/cu. $\mathrm{mm}$. The bone marrow showed an increased number of eosinophil precursors but no other abnormality. Chest radiographs confirmed the presence of a large right pleural effusion. The pleural fluid was serous, contained $3.4 \mathrm{~g} . / 100 \mathrm{ml}$. of protein and $40 \%$ eosinophils but no organisms, and was sterile on culture. Pleural biopsy showed chronic inflammation with numerous eosinophilis but no malignant cells. No tubercle bacilli were recovered from "the sputum, gastric washings, or pleural fluid. No ova or parasites were found in the faeces. An electrocardiogram showed flat or inverted $T$ waves in leads II, III, AVF, V5 and V6. Serum levels of bilirubin, alkaline phosphatase, and globulins were normal. A liver biopsy showed eosinophil infiltration and dilated sinusoids but no other abnormality. Plasma clearance of ${ }^{131}$ I-labelled Rose Bengal was normal, but a liver scan showed many small round areas which did not take up the isotope.

1Present address: Department of Medicine, Royal Postgraduate Medical School. London, W.12
Pleural paracentesis on 8 November was complicated by the alarming development of a pleurobronchial fistula; the patient became severely dyspnoeic with generalized crepitations and cyanosis, and expectorated 1 litre of pleural fluid. The fluid accumulated rapidly and required repeated aspiration of up to 2 litres a day. Cardiac tamponade developed. This was relieved on 28 November by aspiration of sero-sanguineous pericardial fluid which contained numerous eosinophilis and $5.7 \mathrm{~g} . / 100 \mathrm{ml}$. of protein. A pericardial biopsy showed non-specific inflammation.

Treatment was started on 28 November with 60 mg. prednisone a day. Clinical improvement followed, no further aspirations were needed for a month, and the blood eosinophil count fell to normal. As prednisone was withdrawn, the effusions and eosinophilia recurred. Radiological studies showed progressive thickening of the parietal pleura, hilar lymphadenopathy, widening of the intercarinal angle, and paradoxical movement of the right hemidiaphragm. Electrocardiograms showed right ventricular preponderance, generalized flattening of $T$ waves, and electrical alternans.

Cardiotomy was performed on 11 January 1967. Enlarged lymph nodes were palpated anterior to the aorta. The epicardium was studded with fleshy nodules. Pericardial and epicardial biopsies showed the histological appearances of lymphosarcoma.

Following telecobalt therapy to the mediastinum and pericardium, the eosinophil count fell to normal from a maximum level of $11,137 / \mathrm{cu}$. $\mathrm{mm}$. (70\%). On 17 March he died with pneumonia. Permission for necropsy was not obtained.

CASE 2 A previously fit 66-year-old woman was found in October 1965 to have cervical lymphadenopathy and a palpable spleen tip. The white blood cell count was 32,500 , with 24,700 eosinophils/cu. mm. A myelogram showed active eosinophilic leucopoiesis but no other abnormality. An axillary lymph node showed the histological appearance of Hodgkin's 
disease with eosinophil infiltration. There were no other abnormal findings and no other cause of eosinophilia was found. Following radiotherapy to both faciocervical fields the lymphadenopathy and splenomegaly regressed and the eosinophil count fell to $4,600 / \mathrm{cu}$. $\mathrm{mm}$.

In January 1967 submandibular and axillary lymph nodes were enlarged and the blood eosinophil count was $31,000 / \mathrm{cu}$. mm. (90\%). Recent crops of splinter haemorrhages under the fingernails were also noted but remained unexplained. Blood cultures were sterile.

With steroids the eosinophil count fell to 640/ cu. mm., but despite a small maintenance dose of steroids general lymphadenopathy developed two months later and the eosinophil count rose to $38,000 / \mathrm{cu}$. $\mathrm{mm}$.

\section{DISCUSSION}

The combination of a high blood eosinophilia and eosinophilic pleural and pericardial effusions poses an unfamiliar diagnostic problem.

Eosinophilia occurs in 10 to $20 \%$ of patients with lymphosarcoma and Hodgkin's disease (Gall and Mallory, 1942; Rosenberg, Diamond, Jaslowitz, and Craver, 1961) but a high eosinophilia is rare. The highest reported count was 160,000 eosinophilis/cu. mm. (99\%) found in a patient with Hodgkin's disease who also had pleural and pericardial effusions (Major and Leger, 1939).

Pleural effusion is an infrequent presenting feature of lymphosarcoma (Rosenberg et al., 1961). Eosinophilic pleural effusion from any cause is uncommon (Campbell and Webb, 1964), and only one reported case was associated with malignant lymphoma (MacMurray, Katz, and Zimmerman, 1950).

Occasional cases of malignant lymphoma presenting with pericardial effusion have occurred, two with Sternberg-Reed cells and one with eosinophilia in the fluid (Ayerza and Cernich, 1943 ; Merab, Taleb, Saliby, Eliane, Naffah, and Kyriakos, 1959). Eosinophilic pericarditis with pulmonary eosinophilia (Hall, Kozak, and Spink, 1964) and single cases of pericardial effusion and blood eosinophilia with penicillin hypersensitivity (Schoenwetter and Silber, 1965) and with pancreatitis (Mitchell, 1964) have also been recorded.

In each of the present cases corticosteroid therapy resulted in suppression of the eosinophilia but no sustained remission of the disease.

We are grateful to Dr. K. M. Robertson, Dr. G. H. Hall, Dr. D. Mattingly, Dr. A. J. Daley, and Mr. J. L. Griffiths for permission to publish these cases; the haematological studies in the two cases were carried out by Dr. R. D. Hyde and Dr. J. O. P. Edgcumbe, and the histological studies by Dr. G. Stewart Smith and Professor C. V. Harrison, to whom our thanks are also due.

\section{REFERENCES}

Ayerza, L., and Cernich, R. (1943). Granuloma maligno primitivo del pericardio. Rev. Asoc. méd. argent., 57, 981.

Campbell, G. D., and Webb, W. R. (1964). Eosinophilic pleural effusion; a review with the presentation of 7 new cases. Amer. Rev. resp. Dis., 90, 194.

Gall, E. A., and Mallory, T. B. (1942). Malignant lymphoma; a clinicopathologic survey of 618 cases. Amer. J. Path., 18, 381.

Hall, J. W., Kozak, M., and Spink, W. W. (1964). Pulmonary infiltrates, pericarditis and eosinophilia; a unique case of the pulmonary infiltration and eosinophilia syndrome. Amer. J. Med., 36, 135 .

MacMurray, F. G., Katz, S., and Zimmerman, H. J. (1950). Pleuralfluid eosinophilia. New Engl. J. Med., 243, 330 .

Major, R. H., and Leger, L. H. (1939). Marked eosinophilia in Hodgkin's disease. J. Amer. med. Ass., 112, 2601.

Mérab, A., Taleb, N., Saliby, E., Eliane, E., Naffah, M. J., and Kyriakos, S. (1959). Les péricardites Hodgkiniennes. Apropos d'un cas de maladie de Hodgkin révélée par une péricardite avec épanchement et présence de cellules de Sternberg dans le liquide de ponction. Sem. Hôp. Paris, 35, 2590.

Mitchell, C. E. (1964). Relapsing pancreatitis with recurrent pericardial and pleural effusions. Ann. intern. Med., 60, 1047.

Rosenberg, S. A., Diamond, H. D., Jaslowitz, B., and Craver, L. F. (1961). Lymphosarcoma; a review of 1,269 cases. Medicine (Baltimore), 40, 31.

Schoenwetter, A. H., and Silber, E. N. (1965). Penicillin hypersensitivity, acute pericarditis, and eosinophilia. J. Amer. med. Ass., 191, 672. 